\title{
Typologie, caractéristiques structurales et dynamique des faciès forestiers fragiles à Isoberlinia spp. en vue de leur gestion au Togo
}

Marra DOURMA ${ }^{1}$

Kpérkouma WALA ${ }^{1}$

Kudzo Atsu Guelly ${ }^{1}$

Ronald BeLlefontAine ${ }^{2}$

Philippe DeLePORTE 3

Sêmihinva AKPAVI ${ }^{1}$

Komlan BATAWILA ${ }^{1}$

Koffi AKpagana ${ }^{1}$

1 Université de Lomé

Faculté des sciences

Laboratoire de botanique et écologie végétale

BP 1515, Lomé

Togo

${ }^{2}$ Cirad

Umr Agap

Avenue Agropolis

34398 Montpellier Cedex 5

France

${ }^{3}$ Cirad, SupAgro,

Cirad-Inra-Ird-SupAgro

Persyst Upr 80 s/c Umr Eco\&sol

2, place Viala

34060 Montpellier Cedex 2

France

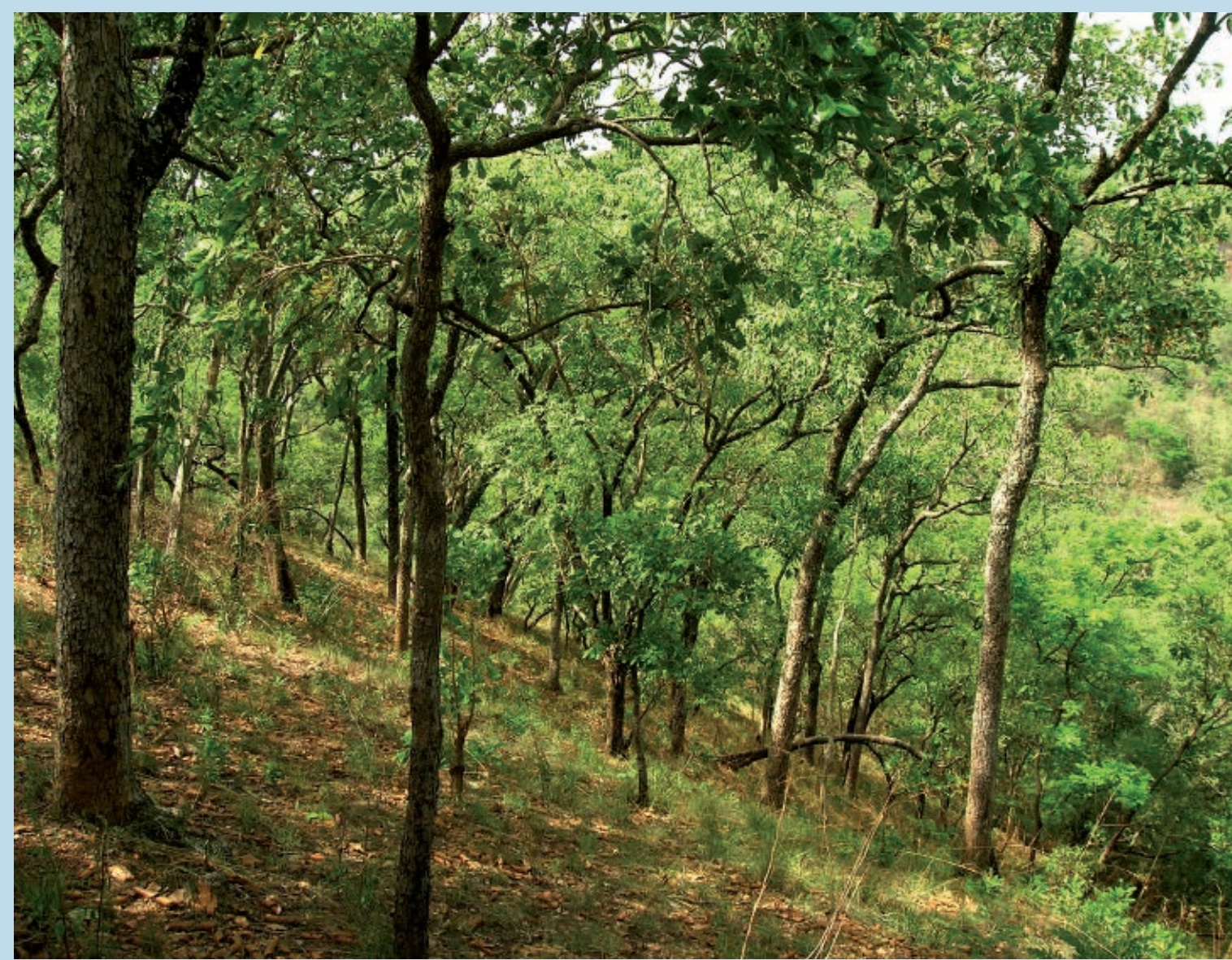

Photo1.

Forêt claire à $I$. doka de haut-versant dans la réserve de Malfa-Kassa.

Photo M. Dourma. 


\section{RÉSUMÉ}

\section{TYPOLOGIE, CARACTÉRISTIQUES STRUCTURALES ET DYNAMIQUE DES FACIĖS FORESTIERS FRAGILES À ISOBERLINIA SPP. EN VUE DE LEUR GESTION AU TOGO}

Les forêts claires à Isoberlinia spp. du domaine soudanien du Togo, rencontrées aussi bien sur les versants que dans les plaines, subissent diverses pressions telles que les défrichements agricoles, l'exploitation du bois de service et de feu ou encore la collecte des produits forestiers non ligneux. Ces forêts ainsi dégradées accentuent la paupérisation des populations riveraines qui en dépendent. Afin de contribuer à leur gestion durable au profit de ces populations locales, il s'avère nécessaire d'analyser l'impact des pressions anthropiques sur la structure de ces forêts. Des inventaires forestiers y ont été conduits. Les travaux ont porté sur 4783 arbres au sein de 225 placeaux de mesures. Plus de cent espèces ligneuses ont été inventoriées comprenant 79 genres et 32 familles, les plus fréquentes étant Isoberlinia doka, Pterocarpus erinaceus, Lannea acida, Isoberlinia tomentosa, Vitellaria paradoxa, Daniellia oliveri et Parkia biglobosa. Les relevés ont permis de déterminer quatre gradients écologiques majeurs : l'humidité, la topographie, le substrat édaphique et l'anthropisation. La classification a identifié neuf faciès forestiers dont quatre en forêts claires, trois en savanes boisées et deux au sein de jachères. En outre, trois composantes structurales ont été définies en analysant la structure diamétrique des neuf faciès. Enfin, il a été aisé de distinguer, d'une part, les forêts naturelles peu perturbées, présentant une stabilité floristique bénéficiant d'un flux de régénération continu, ainsi qu'une régularité de structure, et, d'autre part, les forêts dégradées qui se caractérisent par l'absence de tiges de grande taille et le faible potentiel de régénération. La pression humaine qui s'exerce sur ces forêts fragiles ne peut qu'inciter à reboiser les espaces dégradés, en mettant à profit les techniques de multiplication végétative à faible coût. Reste surtout à atteindre une prise de conscience de la part des populations riveraines pour ce qui a trait à la conservation de ces milieux, et aboutir à une gestion participative.

Mots-clés : forêts claires à Isoberlinia, gestion, structure, typologie, Togo.

\section{ABSTRACT}

\section{TYPOLOGY, STRUCTURAL CHARACTERISTICS AND DYNAMICS OF FRAGILE ISOBERLINIA SPP. WOODLANDS TO SUPPORT THEIR MANAGEMENT IN TOGO}

The open Isoberlinia spp. woodlands found on slopes and valley floors in Togo's Sudanian region are subject to various pressures including clearance for cropfields, cutting for timber or fuelwood and gathering of non-timber forest products. The resulting degradation is worsening poverty among the local people who depend on these forests. To support their sustainable management for the benefit of these local populations, the impacts of human pressures on the forest structure first need to be analysed. The forest inventories conducted covered 4783 trees in 225 study plots. More than one hundred woody species were listed, belonging to 79 genera and 32 families. The most frequent were Isoberlinia doka, Pterocarpus erinaceus, Lannea acida, Isoberlinia tomentosa, Vitellaria paradoxa, Daniellia oliveri and Parkia biglobosa. The surveys identified four main parameters determining ecological gradients: moisture, topography, soil substrate and human influence. Nine woodland facies were identified: four open woodland types, three tree savannah types and two in fallow areas. Three structural components were determined by analysing tree diameter structure for the nine facies. Finally, relatively undisturbed natural forest areas with stable and continuously regenerating flora and regular forest structure were easily distinguished from degraded forest areas characterised by a lack of large trees and low regeneration potential. The human pressures exerted on these fragile woodland areas clearly suggest replanting in the degraded areas, using low-cost multiplication techniques, although raising awareness among the neighbouring populations about the need for conservation remains essential to ensure participatory management.

Keywords: open Isoberlinia forest, management, structure, typology, Togo.
RESUMEN

\section{TIPOLOGÍA, CARACTERÍSTICAS ESTRUCTURALES Y DINÁMICA DE LOS FRÁGILES ARBOLADOS DE ISOBERLINIA SPP. PARA LLEVAR A CABO SU MANEJO EN TOGO}

Los bosques abiertos de Isoberlinia spp. del dominio climático sudanés de Togo, ubicados tanto en pendientes como en llanuras, sufren diversas presiones como desmontes para uso agrícola, extracción de madera o leña y también la recolección de productos forestales no maderables. Estos bosques así degradados acentúan la pauperización de las poblaciones colindantes que dependen de ellos. Para contribuir a un manejo sostenible que beneficie a la población local, es necesario analizar el impacto de las presiones antrópicas en la estructura de dichos bosques. Se realizaron inventarios forestales y los estudios se centraron en 4783 árboles en 225 parcelas de medición. Se han inventariado más de 100 especies leñosas que incluyen 79 géneros y 32 familias, las más frecuentes son Isoberlinia doka, Pterocarpus erinaceus, Lannea acida, Isoberlinia tomentosa, Vitellaria paradoxa, Daniellia oliveri y Parkia biglobosa. Las observaciones permitieron determinar cuatro gradientes ecológicos principales: humedad, topografía, sustrato edáfico y antropización. La clasificación ha identificado nueve facies forestales: cuatro en bosques abiertos, tres en sabanas arboladas y dos en barbechos. Además, al analizar la estructura diamétrica de las nueve facies, se definieron tres componentes estructurales. Por último, fue fácil distinguir, por un lado, los bosques naturales poco alterados y que presentan una estabilidad florística que se beneficia de un flujo de regeneración continuo y de regularidad estructural y, por otro lado, los bosques degradados que se caracterizan por ausencia de tallos de gran tamaño y su bajo potencial de regeneración. La presión humana a la que se ven sometidos estos bosques frágiles sólo puede servir de estímulo para la reforestación de los espacios degradados aprovechando las técnicas de multiplicación vegetativa de bajo costo. Sobre todo hace falta lograr la concienciación por parte de las poblaciones colindantes en todo lo referido a la conservación de estos entornos y conseguir un manejo participativo.

Palabras clave: bosques abiertos de Isoberlinia, manejo, estructura, tipología, Togo. 


\section{Introduction}

La dégradation à un rythme inquiétant des écosystèmes forestiers sous les tropiques a pour conséquence directe la fragmentation des formations végétales. Ceci est aggravé par un certain manque de volonté institutionnelle et l'absence d'outils de gestion adéquats. Il en résulte une réduction considérable de la densité des forêts et l'extinction de certaines espèces (FAO, 2001 ; SWAMINATHAN, 1990).

En Afrique occidentale, de nombreux auteurs (LYKKE, 2000 ; Tente, Sinsin, 2002) ont montré que les activités humaines (agriculture sur brûlis, ranchs et autres parcours, exploitation des mines, urbanisation) ont un impact négatif sur la structure, la composition floristique et la dynamique des forêts naturelles. Au Togo, les forêts claires à Isoberlinia des terroirs villageois et des domaines de l'État connaissent elles aussi une dégradation poussée de leur composante ligneuse, qui constitue la source d'approvisionnement en bois-énergie, en bois de service et en bois d'œuvre (photos $1,2,3,4,5)$ pour les populations locales (Dourma et al., 2009a ; DOURMA, 2011).

La nécessité de sauvegarder les ressources ligneuses, tout en satisfaisant les besoins quotidiens des populations, interpelle aussi bien les décideurs, les chercheurs que les populations et autres acteurs économiques locaux. Toutes ces parties prenantes doivent œuvrer à l'élaboration de politiques de gestion durable des formations végétales, reposant sur des données scientifiques de base bien établies. Les données écologiques, notamment sur la structure des forêts, constituent un indicateur de l'évaluation qualitative et quantitative de tout peuplement forestier (OOterhoorn, Kapelle, 2000). Il s'avère nécessaire de mener des investigations afin de disposer des données scientifiques, outils indispensables pour l'élaboration de plans de gestion durable qui complètent les études précédentes relatives à ces forêts (BATIONO et al., 2005 ; SOKPON et al., 2006 ; Dourma et al., 2006, 2009a, b ; FonTOn et al., 2009 ; DOURMA, 2011).

Cet article analyse la structure des forêts claires à Isoberlinia suivant ses différents faciès et met en exergue leur diversité en termes d'espèces ligneuses et leurs caractéristiques morpho-structurales en relation avec les facteurs écologiques et anthropiques.

\section{Milieu d’étude}

L'étude a été menée dans deux régions (centrale et de la Kara) entre les longitudes $0^{\circ} 20^{\prime}$ et $1^{\circ} 35^{\prime}$ Est et les latitudes $8^{\circ} 15^{\prime}$ et $10^{\circ}$ Nord du Togo (figure 1 ). La zone est limitée au nord par les sous-unités des monts Défalé-Kabyè et au sud par la sous-unité des monts Fazao Malfakassa et Alédjo. Elle appartient au centre régional d'endémisme soudanien (WHITE, 1986). Le relief est irrégulier, compris entre 400 à 500 m d'altitude. Les sols ferrugineux et ferrallitiques tropicaux sont dominants. Les cours d'eau s'écoulent d'est en ouest pour se jeter dans le fleuve Volta au Ghana. Le climat est de type tropical à régime unimodal avec des précipitations de 900 à $1400 \mathrm{~mm} / \mathrm{an}$. Les températures moyennes mensuelles varient de 24,7 à $28,6^{\circ} \mathrm{C}$ et l'humidité relative moyenne de l'air oscille entre 40 et $80 \%$. Les principaux groupes ethniques (Tem, Bassar, Kabyè, Nawdba, Lamba, Tamberma, Agnanga, Peuls) vivent essentiellement des revenus de l'agriculture, de l'élevage, du commerce et de l'artisanat. Ces activités socio-économiques ont des impacts qui influencent négativement la flore et la faune.
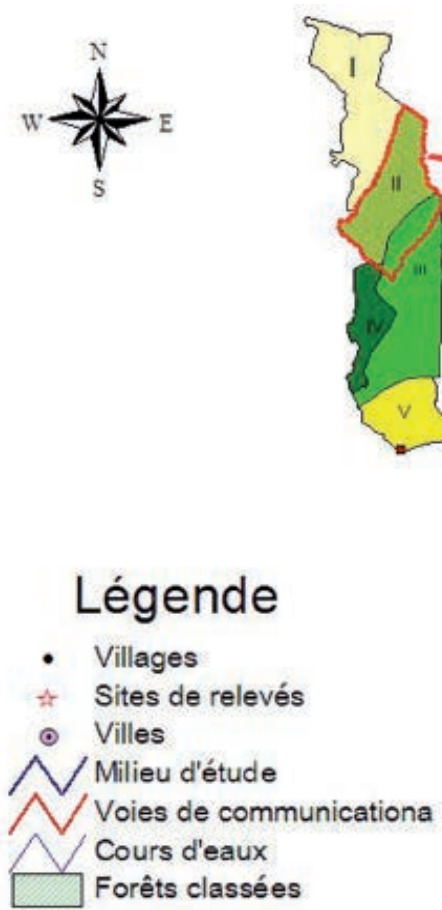

Echelle: $1 / 2500000$ 


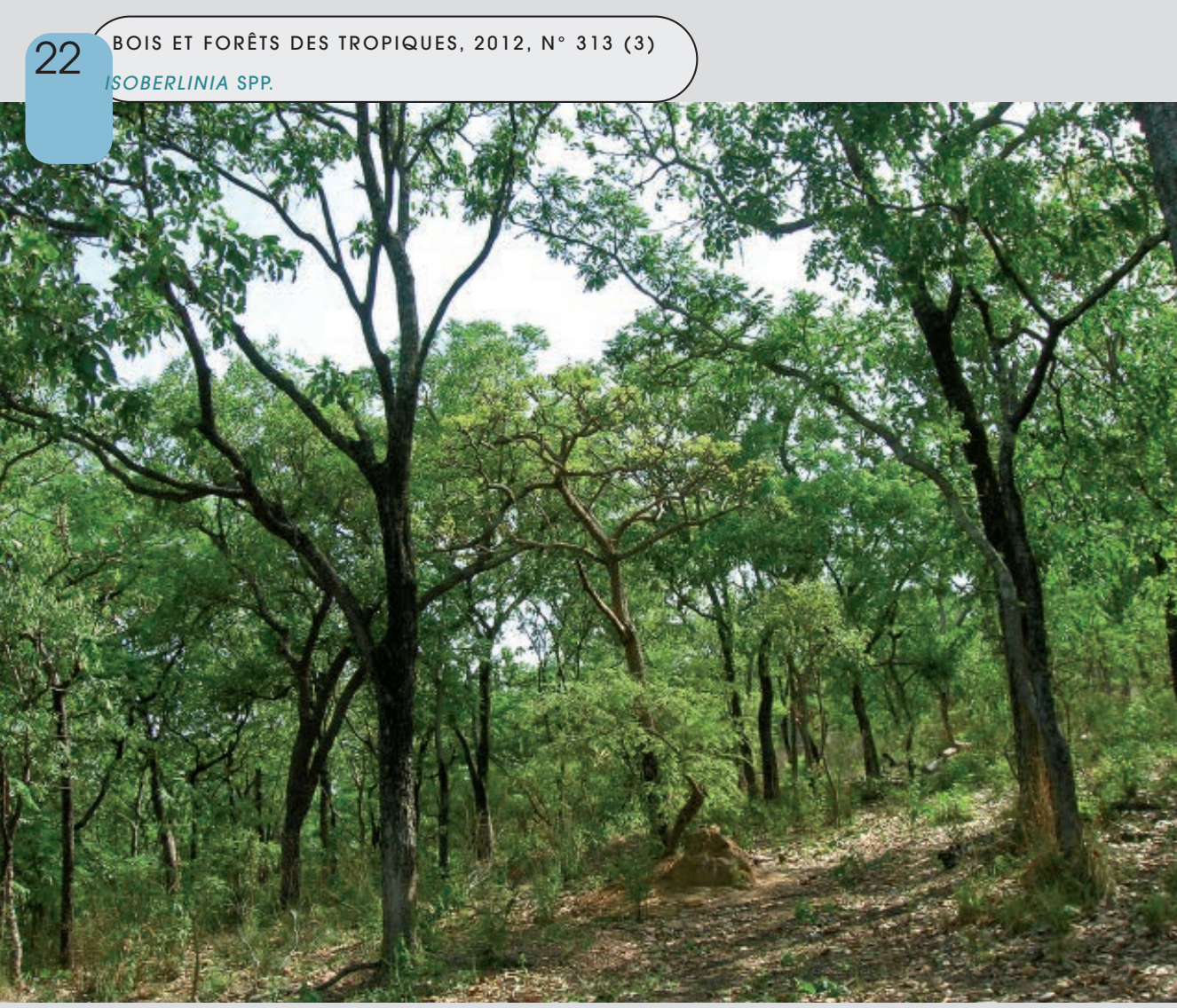

Photo 2.

Piste à l'intérieur d'une forêt claire villageoise à Isoberlinia pour le transport du bois d'œuvre (Agbandi).

Photo M. Dourma.

\section{Traitement de données}

Deux matrices « espèces $x$ relevés » ont été établies en prenant en compte les coefficients d'abondance spécifique et la présence/absence des espèces dans le placeau. Deux autres matrices « descripteurs $x$ relevés » prenant en compte les variables écologiques sont codifiées suivant des grandeurs ordinales et nominales. Ces matrices ont été soumises à des analyses multivariées et au calcul des fréquences. Pour discriminer les différents groupes de relevés qui correspondent à des faciès forestiers sur la base de la composante ligneuse des strates supérieures, la matrice constituée par les abondances des espèces a été soumise à une classification ascendante hiérarchique (Cah). Ci-après, les faciès forestiers discriminés seront nommés par les espèces dominantes autres que Isoberlinia. La matrice précédente couplée à celle des descripteurs écologiques a permis l'ordina-

\section{Méthodologie}

\section{Collecte de données}

Un inventaire forestier des espèces ligneuses a été réalisé dans 33 sites choisis suivant leur variabilité écologique locale après une phase de prospection (figure 1). Au total, 225 placeaux de $400 \mathrm{~m}^{2}$ ont été installés suivant l'approche méthodologique de Braun-BLANQUeT (1932) le long de 68 transects transversaux en partant de la plaine vers les sommets des collines. La longueur du transect est fonction de la taille du site et les placeaux consécutifs sont équidistants de $50 \mathrm{~m}$. La nomenclature utilisée est celle revue et actualisée selon les recommandations de l'« Angiosperm Phylogeny Group » (APG II, 2003).

Le diamètre des tiges à 1,3 m du sol (Dbh), la hauteur et le diamètre du houppier sont mesurés. Les diamètres sont mesurés à l'aide d'un ruban et les hauteurs à l'aide d'un clinomètre. Les tiges juvéniles de Dbh inférieur à $5 \mathrm{~cm}$ sont dénombrées dans la régénération sur trois placettes de $25 \mathrm{~m}^{2}$ installées suivant la diagonale de chaque placeau. Une fiche standard de descripteurs écologiques (hauteur moyenne/recouvrement du peuplement, occupation agricole, topographie, pente, présence de lianes, nature/affleurement de la roche, nature du sol, submersion, drainage superficiel, exposition et gestion actuelle) est remplie en même temps que les relevés floristiques sont réalisés. Les coordonnées géographiques des relevés d'inventaires sont enregistrées à l'aide d'un Gps. tion des placeaux et des descripteurs écologiques par l'analyse canonique de correspondance (Acc). Cette analyse transforme les axes de l'analyse canonique (AC) et se présente comme une combinaison linéaire des variables environnementales relevées sur le terrain ; elle permet la détermination directe des gradients écologiques majeurs. Les variables écologiques ont été codifiées suivant des échelles ordinales à partir de 1 ou des échelles nominales en présence/absence. Le choix de ces analyses multivariées est guidé par leur interprétabilité écologique (HILL, GAUCH, 1980). L'Acc a été réalisée grâce au logiciel Canoco version 4 (Ter BraAk, Smilauer, 1998) et la classification a été effectuée grâce au logiciel Statistica 6.0.

L'indice de valeur d'importance des espèces (IVi), mis au point par COTTAM, CURTIS (1956), caractérise la place qu'occupe chaque espèce par rapport à l'ensemble des espèces d'une végétation. Il a été calculé pour évaluer la prépondérance spécifique (KouAmÉ, 1998) suivant la formule :

\section{Ivi $=$ FREQesp + DENSesp + DOMesp,}

avec :

- FREQesp, la fréquence relative d'une espèce ; c'est le rapport de sa fréquence spécifique (nombre de placettes dans lesquelles elle est présente) au total des fréquences spécifiques ; - DENSesp, la densité relative d'une espèce ; c'est le rapport de sa densité absolue au total des densités absolues ;

- DOMesp, la dominance relative d'une espèce ; c'est le quotient de son aire basale (surface terrière) par le total des aires basales des espèces. 


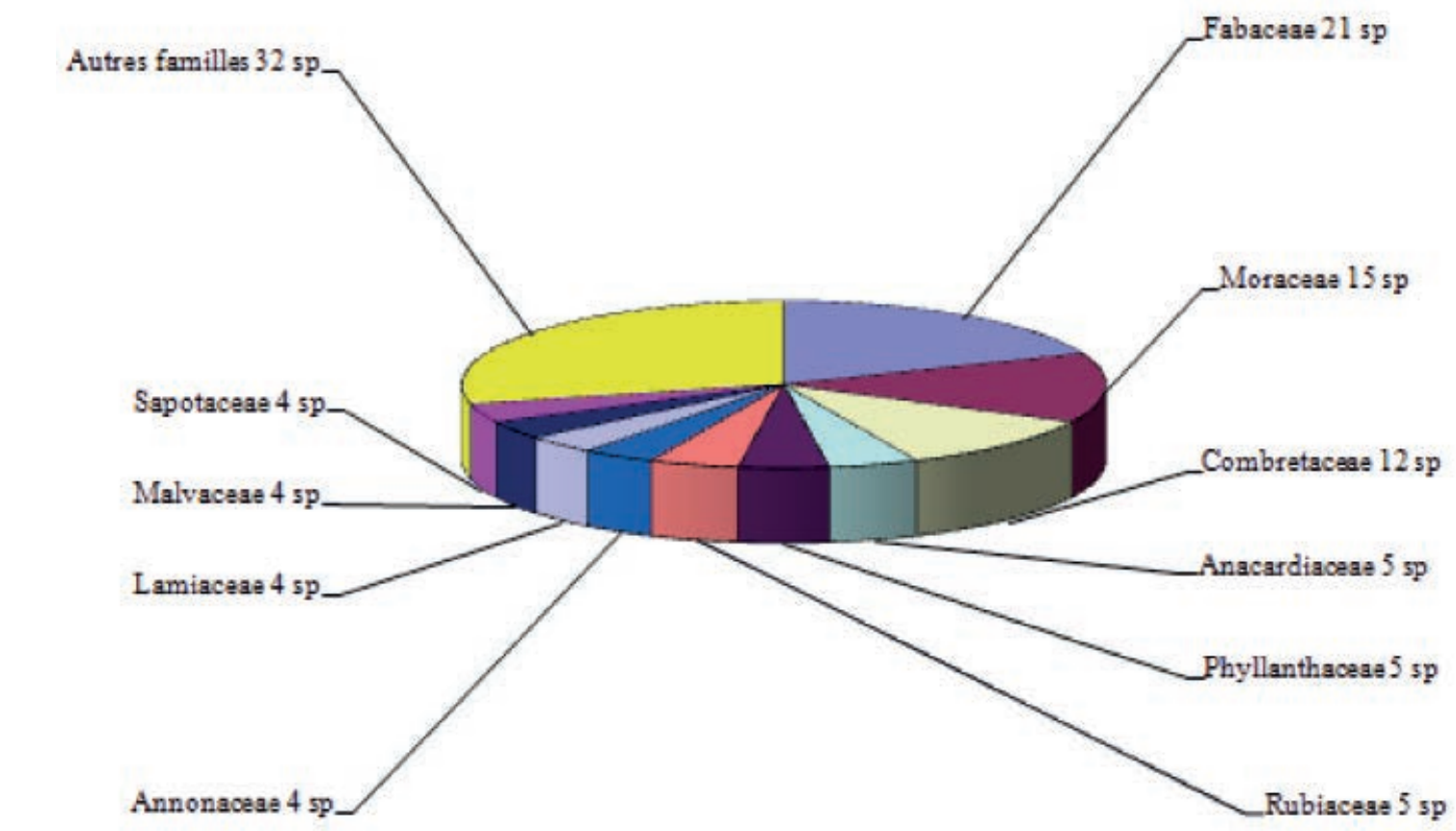

Figure 2.

Spectre des familles d'espèces ligneuses ( $\mathrm{sp}$ : espèce) des forêts à Isoberlinia étudiées.

Tableau I.

Espèces ligneuses prépondérantes des forêts à Isoberlinia spp.

\begin{tabular}{|c|c|c|c|c|}
\hline Espèces & Fréquence relative & Densité relative & Dominance relative & Ivi \\
\hline Isoberlinia doka & 27,1 & 25,4 & 28,4 & 80,9 \\
\hline Pterocarpus erinaceus & 18,7 & 15,5 & 27,1 & 61,3 \\
\hline Lannea acida & 12,9 & 12,2 & 24,9 & 50,1 \\
\hline Isoberlinia tomentosa & 10,0 & 12,1 & 24,3 & 46,4 \\
\hline Vitellaria paradoxa & 12,4 & 9,5 & 18,6 & 40,5 \\
\hline Daniellia oliveri & 11,7 & 11,3 & 17,2 & 40,2 \\
\hline Parkia biglobosa & 7,6 & 7,5 & 8,9 & 24,0 \\
\hline Monotes kerstingii & 7,5 & 6,2 & 7,3 & 21,0 \\
\hline Cussonia kirkii & 7,1 & 7,5 & 5,9 & 20,5 \\
\hline Uapaca togoensis & 6,9 & 5,5 & 7,9 & 20,3 \\
\hline Afzelia africana & 6,8 & 6,5 & 5,9 & 19,2 \\
\hline Hannoa ondulata & 6,8 & 7,1 & 4,8 & 18,7 \\
\hline Pericopsis laxiflora & 6,2 & 6,5 & 4,9 & 17,4 \\
\hline Parinari curatellifolia & 5,9 & 2,7 & 3,6 & 12,2 \\
\hline Burkea africana & 4,3 & 3,7 & 3,6 & 11,6 \\
\hline Entada africana & 4,2 & 2,7 & 3,6 & 10,5 \\
\hline Autres & 68,6 & 12,3 & 14,2 & 95,1 \\
\hline
\end{tabular}



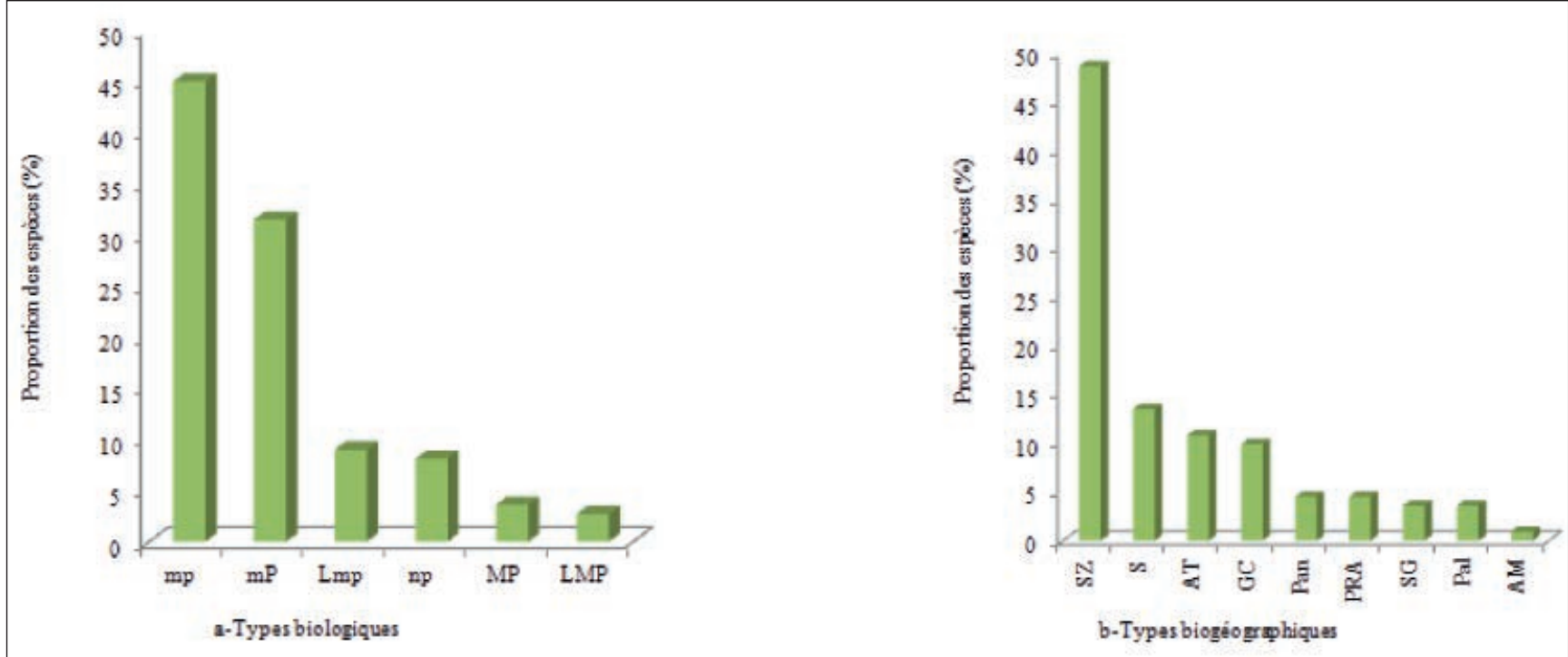

Figure 3.

Spectres biologiques (a) et phytogéographiques (b) bruts des ligneux des forêts à Isoberlinia étudiées.

(mp : microphanérophytes ; $\mathrm{mP}$ : mésophanérophytes, $\mathrm{np}$ : nanophanérophytes ;

MP : mégaphanérophytes ; LMP : lianes mégaphanérophytes ; SZ : soudano-zambézien ;

AT : afrotropicales ; SG : soudano-guinéennes ; Pan : pantropicales ; S : élément-base soudanien ;

GC : guinéo-congolaises ; Pal : paléotropicales ; PRA : plurirégionales africaines ; $A M$ : afro-malgache).

\section{Typologie des peuplements à Isoberlinia sur la base des espèces ligneuses}

Deux groupes de relevés se dégagent sur la base de la composante ligneuse des forêts claires par la Cah globale : les relevés des monts, et des buttes et plateaux. Ils correspondent à quatre composantes au seuil de $35 \%$ de dissimilitude.

L'analyse partielle des relevés des monts révèle deux faciès : les relevés PA1 ou le type à Afzelia africana et Parinari curatellifolia et les relevés PA2 ou le type à Monotes kerstingii et Burkea africana (figure 4a).

L'analyse partielle des relevés du groupe des buttes et plateaux discrimine deux faciès : les relevés de mi-versant (PB) (figure 4a) ou le type à Hexalobus monopetalus et Detarium microcarpum.

Les relevés de bas-versant, sur les buttes argileuses et cuirassées (PC), sont divisés au seuil de $37 \%$ d'agrégation en trois faciès : le type à Uapaca togoensis (PC1), le type à Piliostigma thonningii et Terminalia laxiflora (PC2), le type à Daniellia oliveri et Pteleopsis suberosa (PC3) (figure 4b).

Les relevés des plaines (PD) sont divisés au seuil de $78 \%$ en trois faciès : le type à Pterocarpus erinaceus et Hannoa undulata (PD1), le type à Vitellaria paradoxa et Parkia biglobosa (PD2) et le type à Maranthes polyandra (PD3) (figure 4c).

L'Acc a permis de montrer que trois gradients majeurs (l'humidité, la topographie et le substrat édaphique) ont permis la distribution des relevés. Suivant la toposéquence du bas vers le haut de versant, les forêts les plus fermées se dégagent des plus ouvertes selon le gradient d'humidité, tandis que le gradient édaphique sépare les peuplements naturels sur sols profonds à gravillons fins de ceux sur sols squelettiques à gros gravillons (figure 5).

Le test de Monte Carlo montre que neuf descripteurs écologiques codifiés suivant des grandeurs nominales sont les mieux corrélés avec les axes canoniques 1 et $2(p=0,002)$. Le plan 1-2 exprime $65,2 \%$ de la variance des relations espèces-descripteurs écologiques. Les descripteurs «zones classées à statut de protection ", " affleurement rocheux », « traces de chasse », " pâturage », « cultures » sont les mieux corrélés suivant l'axe 1. L'opposition entre les descripteurs « zone classée » et " pâturage » s'explique par le fait que les sites protégés sont peu parcourus par le bétail. L'opposition observée entre les descripteurs " affleurement rocheux », « chasse » et « cultures » peut s'expliquer par le fait que les terres à relief irrégulier peu propices à l'agriculture font partie des zones classées souvent objet de chasse incontrôlée et frauduleuse. Les descripteurs les mieux corrélés « induration du sol », " présence de gravillons » sont opposés aux descripteurs «emplacement de la fabrication de charbon de bois ", " passage de feux de brousse » suivant l'axe 2. Dans ce cas, des tiges de faible diamètre sont observées sur sols indurés, délaissés par l'agriculture (figure 5a).

Le test de Monte Carlo montre que quatorze descripteurs écologiques codifiés suivant des grandeurs ordinales sont très bien corrélés avec le plan 1-2 $(p=0,032)$ qui exprime $48,3 \%$ de la variance de la relation espèces-descripteurs écologiques.

Les descripteurs "type de végétation », "drainage superficiel du sol », "présence de lianes », "pente », " distance séparant les relevés des champs et des villages", «fermeture de la formation » sont corrélés positivement avec l'axe 1. Par contre, les descripteurs « exposition du site ", " taux d'occupation de cultures/jachères ", " recouvrement de la strate arborée » sont bien corrélés, mais négativement avec l'axe 1. Suivant l'axe 2, le descripteur « humidité du sol » corrélé positivement s’oppose aux descripteurs « taille » et « recouvrement moyen de la strate herbacée », qui sont quant à eux bien corrélés, mais négativement (figure 5b). 


\section{Caractéristiques écologiques et floristiques des peuplements}
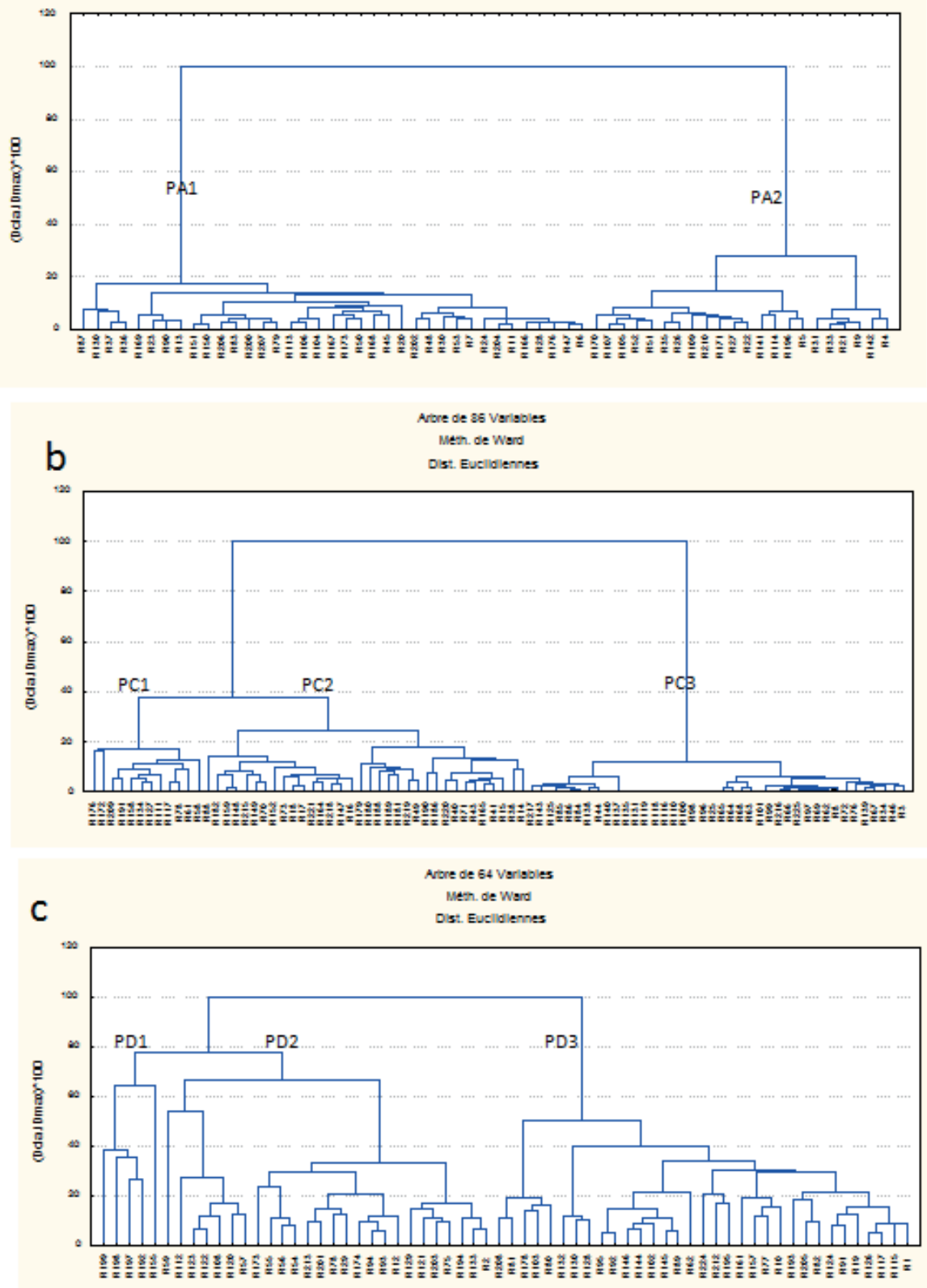

Figure 4.

Classification ascendante hiérarchique partielle des relevés des trois groupes compacts de l'analyse globale. Faciès forestiers à : Afzelia africana et Parinari curatellifolia (PA1), Monotes kerstingii et Burkea africana (PA2), Uapaca togoensis (PC1), Piliostigma thonningii et Terminalia laxiflora (PC2), Daniellia oliveri et Pteleopsis suberosa (PC3), Pterocarpus erinaceus et Hannoa undulata (PD1), Vitellaria paradoxa et Parkia biglobosa (PD2), Maranthes polyandra (PD3)).

Les peuplements à Isoberlinia étudiés présentent trois types physionomiques principaux : les forêts claires qui constituent le type dominant, les savanes boisées résultant d'une dégradation de la forêt claire et les jachères qui sont d'anciennes forêts claires en reconstitution après une période de mises en culture. Ces différents faciès présentent des caractéristiques structurales bien spécifiques (tableau II).

\section{Type à Afzelia africana et Parinari curatellifolia (PA1)}

Ces forêts claires se développent sur les sols ferrugineux tropicaux indurés en profondeur à texture argileuse. Ces sols peu profonds montrent des gravillons et des affleurements de roches quartzitiques. Ces forêts affectionnent les versants des collines aux pentes faibles où le substrat édaphique est relativement pauvre en matière organique. L'humidité relative du sol est faible à cause du contraste pédologique et de la position topographique. Ces peuplements des localités d'Agaradè, Soudou, Kidéoudé, Amaoudè-Mô, Agbang, Folo, Bagban et Yaka sont fréquemment le siège des feux de brousse en saison sèche.

\section{Type à Monotes kerstingii et Burkea africana (PA2)}

Ce type de forêts claires se retrouve sur des replats de haut-versant aux sols ferrugineux tropicaux indurés profonds à Alédjo, Kolina, Fazao-Malfakassa, Dako, Tchatchaminadè et Lama-Tessi.

\section{Type à Hexalobus monopetalus et Detarium microcarpum (PB)}

Ce sont des forêts claires de mi-versant des monts Tchaoudjo et Assoli, situées précisément à Djamdè, Wiya, Agaradè, Bafilo sur des sols ferrugineux indurés peu et moyennement $(20-60 \mathrm{~cm})$ profonds.

\section{Type à Uapaca togoensis (PC1)}

Ce sont des forêts claires de plateau et de plaine aux sols ferrugineux tropicaux lessivés, indurés, superficiels, à texture argilosableuse sur des zones d'escarpement identifiées à Agbandi, Téhéza, Affem-kabyè et Alédjo-Kadara. 



Figure 5.

Ordination des relevés et des variables écologiques « nominales » (a)

et « ordinales » (b) dans le plan factoriel des axes 1 et 2 de la Cca.

\section{Type à Piliostigma thonningii et Terminalia laxiflora (PC2)}

Ce sont des savanes de plaine présentes sur divers types de sols indurés profonds retrouvés à Alédjo-Mô, Djamdè, Agaradè, Broukou, Kadjalla et Aléhéridè. Elles sont actuellement très sollicitées par les populations locales pour leurs ressources ligneuses, d'où la modification du type physionomique de départ (photo 6).

\section{Type à Daniellia oliveri et Pteleopsis suberosa (PC3)}

Ce sont des savanes boisées de bas-versant et de butte aux pentes faibles avec un tapis herbacé développé et continu, résultant de la dégradation des forêts claires. Elles se retrouvent sur des sols argilo-sableux indurés peu profonds avec ou sans affleurements de roches à Agbang, Téméré, Agbandi, Téhéza, Pagala, Fazao et Baga.

\section{Type à Pterocarpus erinaceus et Hannoa undulata (PD1)}

Ce sont des forêts claires sur sols ferrugineux latéritiques, indurés, à affleurements de blocs de cuirasse, très peu profonds, qui sont localisées à Bafilo, Alédjo, Aléhéridè et Lama-Tessi. Elles sont sollicitées pour la fabrication de charbon de bois et pour le bois de feu.

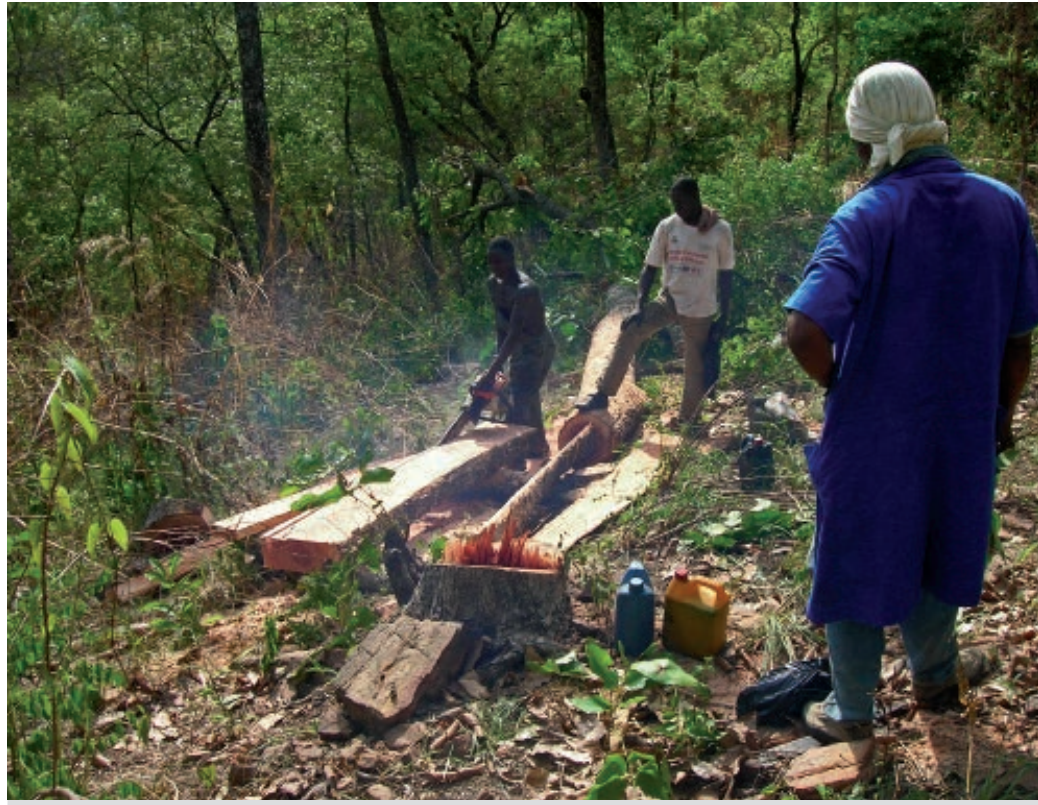

Photo 4.

Exploitation artisanale du bois d'œuvre d'un I. doka (Lama-tessi).

Photo M. Dourma. 
Tableau II.

Résultats synthétiques et analytiques des neuf types de faciès à Isoberlinia discriminés.

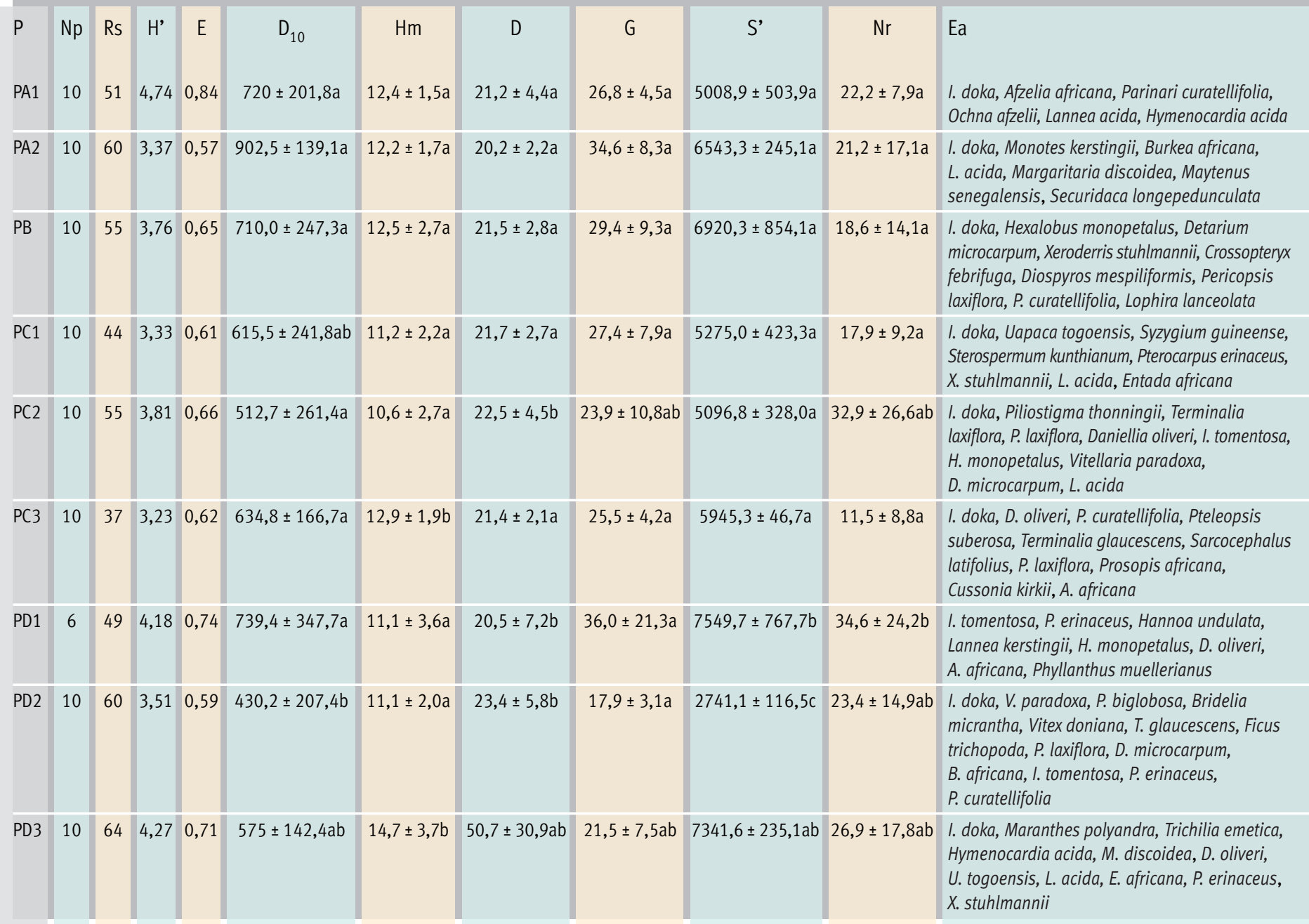

$\mathrm{P}$ : types de faciès; $\mathrm{Np}:$ nombre de placeaux; Rs : richesse spécifique moyenne ; H’ : indice de Shannon (bits) ; E : équitabilité de Pielou ; $\mathrm{D}_{10}$ : densité (tiges/ha); Hm: hauteur moyenne $(\mathrm{m}) ; \mathrm{D}$ : diamètre à $\mathrm{Dbh}>10 \mathrm{~cm}(\mathrm{~cm}) ; \mathrm{G}$ : surface terrière $\left(\mathrm{m}^{2} / \mathrm{ha}\right)$;

$\mathrm{S}^{\prime}$ : recouvrement du houppier $\left(\mathrm{m}^{2} / \mathrm{ha}\right) ; \mathrm{Nr}$ : nombre de régénérations toutes espèces confondues ; Ea : espèces abondantes.

Groupes de même lettre : absence de différence significative.

\section{Type à Vitellaria paradoxa et Parkia biglobosa (PD2)}

Ce sont d'anciennes jachères reconstituées des sols ferrugineux tropicaux non indurés des plateaux et des plaines, propices à l'agriculture et souvent pâturées (photo 7). Les sols sont profonds, à texture argileuse avec deux variantes : il s'agit des sols à concrétions grossières de gneiss et de micaschistes retrouvés à Lama-Tessi, Wiya, Broukou, Agbandi, Téhéza et des sols à quartzites et gneiss de Fazao, Aléhéridè, Pagala, Kidéoudè et Kolina.

\section{Type à Maranthes polyandra (PD3)}

Ce sont des forêts claires de vallée et de plaine qui se caractérisent par des grands arbres, actuellement exploités comme bois d'œuvre. Ce type de peuplements se développe sur des sols ferrugineux tropicaux non indurés, profonds (plus de $60 \mathrm{~cm}$ ) (photos 8, 9). Ces sols argileux profonds aux gravillons fins et sans affleurement rocheux ont une texture argilo-limoneuse. Ils se localisent à Boulowou, Fazao, Tchapossi, Sourkou, Kabou, Pagala, Agbandi et Tabendè sur de faibles pentes, compte tenu de leur position topographique.

\section{Impact anthropique sur les caractéristiques structurales des faciès et sur leur dynamique de régénération}

Les caractéristiques structurales montrent des valeurs très variables suivant les différents faciès. Ces paramètres dépendent du mode de gestion actuel appliqué à ces forêts par les populations riveraines. La densité des tiges est relativement élevée dans les groupes PA1, PA2, PB et PD1 avec des valeurs supérieurs à 700 tiges/ha. Les hauteurs et les diamètres moyens les plus importants sont enregistrés dans le groupe PD3 qui présente la surface terrière moyenne la 
plus petite avec un grand recouvrement des houppiers. Ces valeurs sont significativement différentes au seuil de $5 \%$ suivant le test de Newman-Keuls pour la densité $\left(p=0,0310^{-3}\right)$, la hauteur $(p=0,01)$, le diamètre $\left(p=0,0210^{-5}\right)$, la surface terrière $\left(p=0,0310^{-4}\right)$ et le recouvrement des houppiers $\left(p=0,0410^{-4}\right)$.

La répartition des arbres inventoriés par classes de diamètre montre trois groupes allométriques (figure 6). Une distribution en « $L$ » est observable pour les forêts naturelles où l'influence humaine est faible. La distribution des arbres en classes de diamètre ou de hauteur montre une prédominance des tiges de petits diamètres qui diminue progressivement vers les classes de grands diamètres, ce qui prouve une dynamique régulière des forêts (PA1, PA2, PB, PC3, $P D 2)$. Un deuxième groupe de distribution est caractérisé par une baisse considérable des tiges de basses classes et l'absence de grandes classes de diamètre (PC1, PC2, PD1). Ce sont des forêts perturbées par l'exploitation artisanale et frauduleuse de bois d'œuvre et de bois-énergie. Sur ces sites, les régénérations observées en l'absence des semenciers sont essentiellement des rejets de souche et des drageons. Enfin, le troisième groupe montre une distribution erratique avec très peu de tiges quelles que soient les classes de diamètre considérées. Ce sont des forêts très dégradées où les feux de végétation représentent une pratique courante lors des défrichements agricoles. Les régénérations enregistrées seront décimées par les feux. Les valeurs du nombre moyen de régénérations suivant le test de Newman-Keuls au seuil de $5 \%(p=0,001)$ montrent des différences significatives avec des écarts-types et des erreurs-types très dispersés.

\section{Discussion}

\section{Diversité des faciès forestiers à Isoberlinia}

Cette étude vient compléter les données existantes sur les forêts claires à Isoberlinia dans la sous-région (BATIONO et al., 2005 ; SOKPON et al., 2006 ; DourMA et al., 2006, 2009a, b ; FonTOn et al., 2009 ; Dourma, 2011). En effet, WATSON et DALLWITZ (1993) signalent leur présence en bandes quasi continues du Mali jusqu'à l'Ouganda entre $4^{\circ}$ et $13^{\circ}$ de latitude Nord dans le domaine soudanien qui correspondent à leurs homologues du domaine zambézien situés entre $5^{\circ}$ et $22^{\circ}$ de latitude Sud dans les régions boisées à « miombos ». Les types décrits sont semblables aux forêts claires homologues étudiées dans le centre régional d'endémisme soudanien (WHITE, 1986 ; SINSIN, 2001 ; Houinato, 2001 ; AKò̇gninou, 2004 ; Оuoba, 2006 ; OuÉdraogo, 2006) et au «Monotes kerstingii open woodland » décrit au Nord du Nigeria (KeRshaw, 1968).

Les types à $M$. kerstingii et $B$. africana, à $U$. togoensis, à $P$. erinaceus et $H$. undulata, qui se développent sur les sols ferrugineux tropicaux indurés peu profonds à texture argileuse où affleurent des roches, sont similaires à ceux décrits dans le groupement de forêts claires à $I$. doka et M. kerstingii au Bénin (Houınato, 2001) et au Burkina Faso (OUоBA, 2006). Les types à $M$. kerstingii et $B$. africana, à $U$.

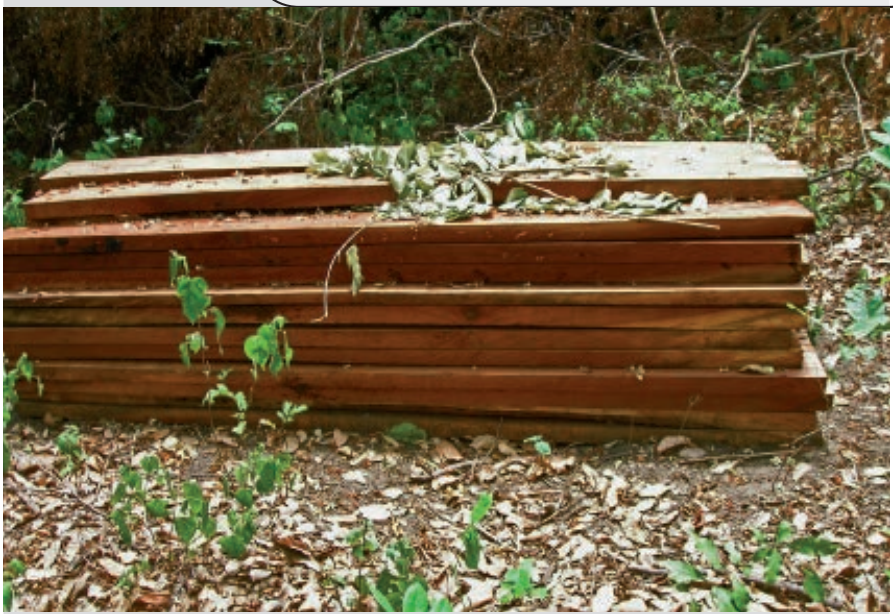

Photo 5 a.

Lieu d'entreposage de planches de Pterocarpus erinaceus en forêt claire à Isoberlinia (Lama-Téssi).

Photo M. Dourma.

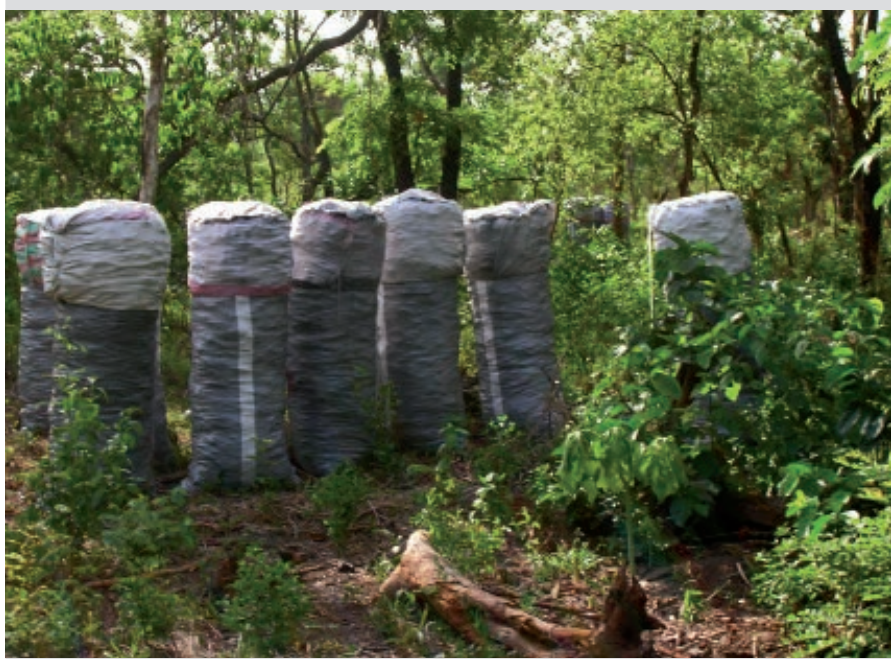

Photo 5b.

Stockage de sacs de charbon de bois dans une savane boisée à Isoberlinia (Mô).

Photo M. Dourma.

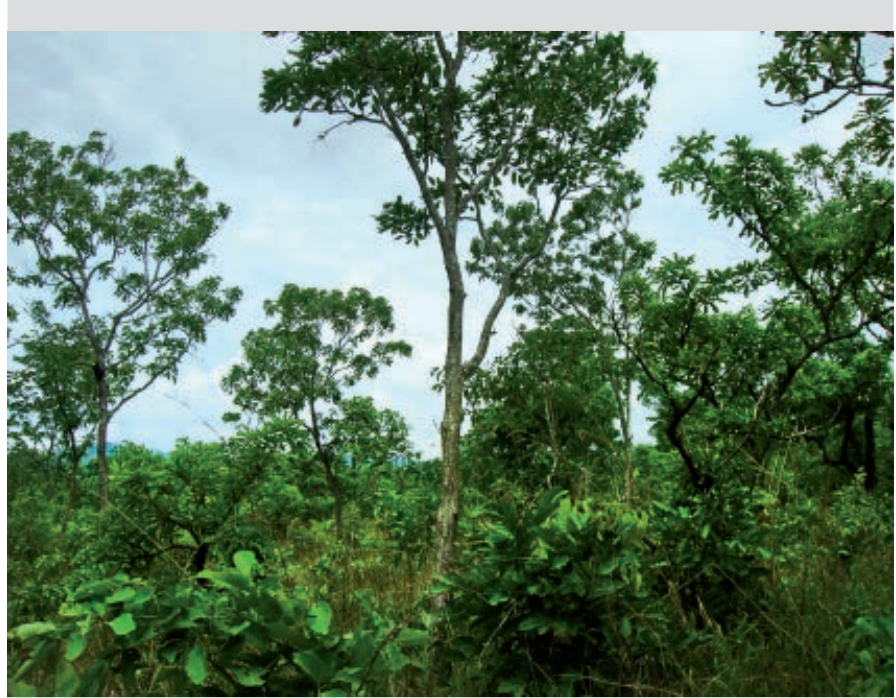

Photo 6.

Savane arborée à Isoberlinia et Vitellaria en saison des pluies (Broukou).

Photo M. Dourma. 
grande importance économique pour les populations locales (Khaya, Pterocarpus, Afzelia, etc.), qui constituent une source de fourrage en saison sèche.

Les peuplements à Isoberlinia sont des formations mixtes, ligneuses et herbeuses, de hauteur dominante variant entre 12 et $18 \mathrm{~m}$ et à couvert ligneux de $50 \%$. Cette structure est régie par la topographie, le substrat édaphique et l'humidité relative du sol. Toutefois, les modes de défrichement agricole, les feux de végétation, les pâturages, la fabrication du charbon de bois, l'exploitation des ressources ligneuses et la récolte des produits forestiers non ligneux modifient la structure et influencent la diversité des peuplements, comme le précisent les travaux de WITKOWSKI et O'CONNOR (1996).

Les valeurs des indices (Shannon, Pielou) relativement plus élevées indiquent que, malgré la forte pression humaine, quelques reliques de peuplements naturels existent encore. Ces résultats corroborent les études en forêt classée de Niangoloko au Burkina Faso (OuOBA, 2006), contrairement à ceux aux faibles indices signalés dans les groupements homologues au Bénin (SINSIN, 2001 ; AKoÈGNINOU, 2004).

La densité, la hauteur, le diamètre, la surface terrière, le recouvrement montrent deux tendances:

- les bas-versants aux sols profonds peu indurés, où l'humidité relative du sol est importante, affichent pour ces cinq paramètres des valeurs élevées, conséquence des bonnes conditions de développement pour les végétaux ; ceci est prouvé par la forte représentation de grands arbres observée, avec une distribution stable des tiges suivant les classes de diamètre assimilables à des classes d'âge, comme le suggèrent d'autres études (FABER-LANGENDOEN, GENTRY, 1991) ;

- sur les hauts de versant aux sols indurés peu profonds, la baisse de l'humidité relative du sol défavorise le développement des végétaux, d'où les faibles valeurs pour ces cinq paramètres.

Ces fortes valeurs enregistrées sur les bas-versants sont dues à l'approche méthodologique et à la prise en compte de peuplements anthropisés.

En ce qui concerne la viabilité des régénérations, le développement des rejets de souche et des drageons est probablement entravé par les parcours de troupeaux, les défrichements et les feux de brousse. En général, le potentiel de régénération des ligneux est observable dans les différents sites, mais la reconstitution des peuplements anthropisés est mauvaise en l'absence de la mise en défens contre les troupeaux et les feux.

La distribution agrégative d'/soberlinia résulterait du mode de dissémination autochore des graines et de sa grande aptitude au drageonnage (DouRMA et al., 2006). La dissémination des graines dans le voisinage immédiat des semenciers, couplée au drageonnage, favorise la colonisation de l'espace, comme précédemment signalé dans la forêt de Nazinon au Burkina Faso (BATıono et al., 2005). En ce qui concerne le développement des drageons sous les houppiers des Isoberlina, zone à faible luminosité, BELLEFONTAINE (2005) émet l'hypothèse que les drageons non autonomes (sans racines individuelles) ne suivent pas une " stratégie» de régénération, mais colonisent l'espace en vue d'augmenter la surface photosynthétique de l'arbre-mère.

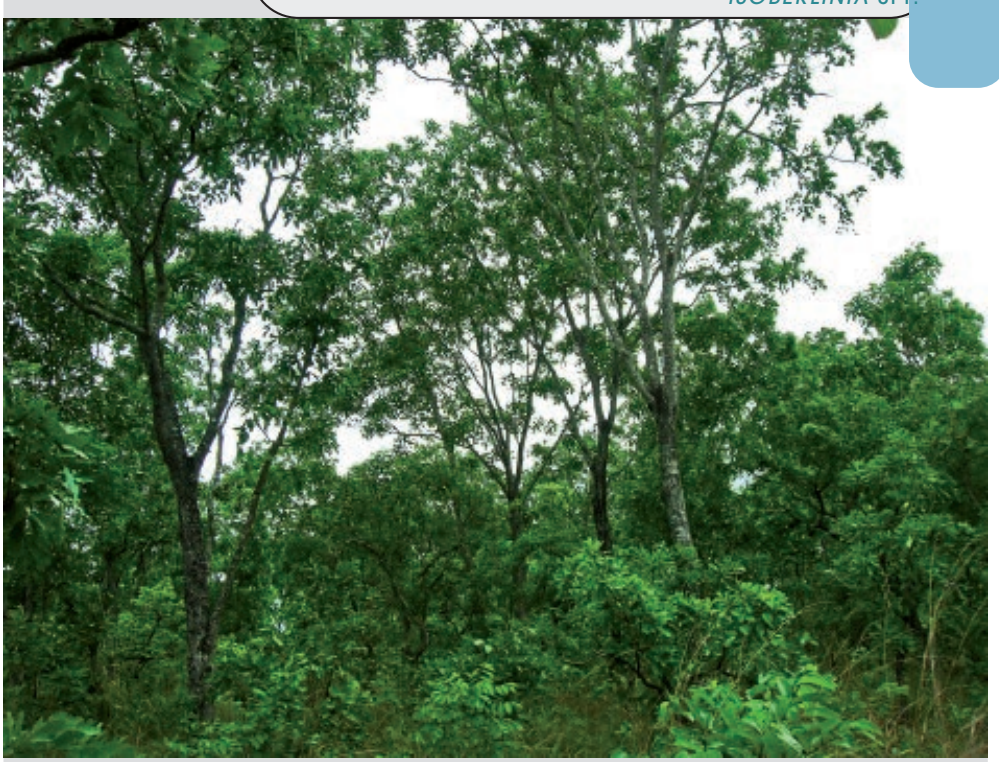

Photo 8.

Peuplement de plaine à Isoberlinia de gros diamètres (Sourkou).

Photo M. Dourma.

\section{Conclusion}

La présente étude a permis de mettre en évidence les diverses pressions anthropiques exercées sur les faciès forestiers à Isoberlinia spp. au Togo. L'exploitation frauduleuse et excessive du bois, couplée à la pression agricole et pastorale et à celle de la fabrication de charbon de bois (prenant une ampleur considérable), compromet la pérennité de ces faciès forestiers. Cette exploitation s'avère alarmante, car elle provoque des changements structuraux qui portent sur de jeunes peuplements n'ayant pas atteint leur état d'équilibre. Cette modification physionomique aux dépens des espèces ligneuses emblématiques n'est pas sans conséquences non seulement vis-à-vis de la biodiversité des forêts claires, mais aussi pour le bien-être des populations riveraines. C'est pourquoi l'évolution de la structure des faciès forestiers suite aux différentes perturbations de l'environnement a été analysée. Les résultats ainsi obtenus complètent les données existantes qui serviront de référence pour l'élaboration de fiches techniques en vue d'une gestion rationnelle de ces forêts fragilisées. L'aspect majeur qui émerge de l'étude est, sans conteste, la facilité avec laquelle ces deux Isoberlinia peuvent se régénérer par semis, drageons et rejets de souche. Cette aptitude constitue une voie favorable pour l'enrichissement de ces forêts claires. Même si leur devenir est davantage du ressort des décideurs politiques, des services gestionnaires et des acteurs économiques que de celui des scientifiques, il est pourtant nécessaire que la recherche soit poursuivie pour chaque type spécifique d'écosystème végétal. De fait, l'absence de gestion de ces forêts naturelles, mettant en péril leur pérennité, impose une prise de conscience collective pour déclencher des mesures concrètes de protection et de conservation, ce qui passe par une sensibilisation de masse et par la mise en place d'un cadre juridique, législatif et réglementaire, protecteur des ressources naturelles du pays. 


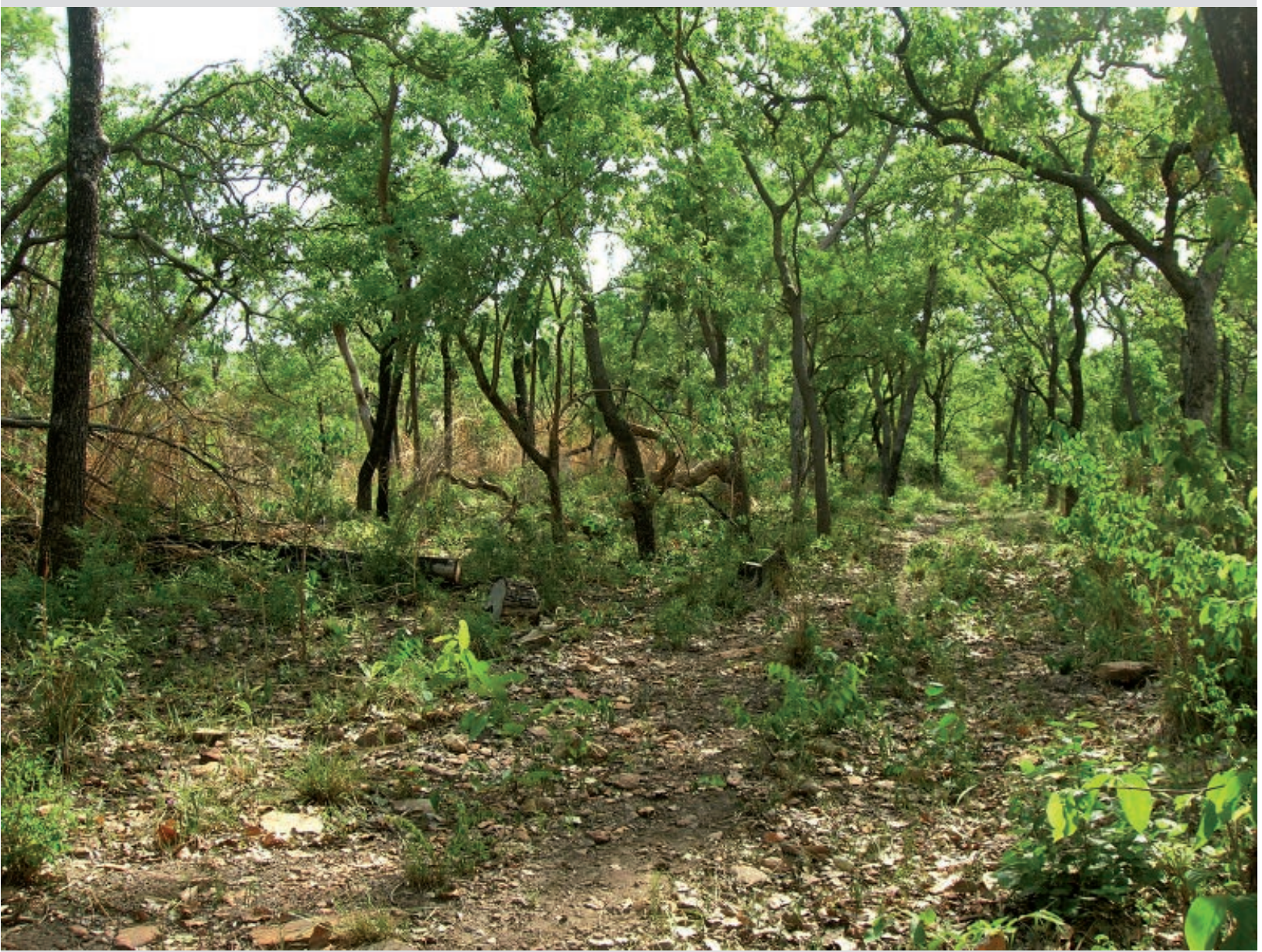

Photo 9.

Traces de pâturage de bœufs dans un peuplement à Isoberlinia. Photo M. Dourma.

\section{Remerciements}

Les auteurs remercient la Fis (Fondation internationale pour la science), le Comstech (Comité permanent pour la coopération scientifique et technologique de l'Organisation de la Conférence islamique), Afornet (African Forest Research Network) et l'Oibt (Organisation internationale des bois tropicaux) pour leur soutien financier.

\section{Références bibliographiques}

ADJONOU K., ALI N., KOKUTSE A. D., NOVIGNO S. K., KOKOU K., 2010. Étude de la dynamique des peuplements naturels de Pterocarpus erinaceus Poir. (Fabaceae) surexploité au Togo. Bois et Forêts des Tropiques, 306 (4) : 45-55.

AKOĖGNINOU A., 2004. Recherches botaniques et écologiques sur les forêts actuelles au Bénin. Thèse d'État, Université de Cocody-Abidjan, Côte d'Ivoire, 326 p.

APG II, 2003. An update of the Angiosperms Phylogeny Group classification for the orders and families of flowering plants: APG II. Botanical Journal of Linnean Society, 141: 399-436.

BATIONO B. A., OUÉDRAOGO S. J., PALLO F., BOUSSIM I. J., 2005. Régénération naturelle d'Isoberlinia doka Craib \& Stapf dans la forêt classée de Nazinon (Burkina Faso). Cahiers d'Études et de Recherches Francophones/Agricultures, 14 (3) : 297-304. 
BELLEFONTAINE R., 2005. Pour de nombreux ligneux, la reproduction sexuée n'est pas la seule voie : analyse de 875 cas. Sécheresse (revue électronique), n $3 E$ (60 p.), décembre 2005, 16 (4) : 315-317.

BRAUN-BLANQUET J., 1932. Plant Sociology. New York et Londres, Mac Graw-Hill, 330 p.

COTTAM G., CURTIS J. T., 1956. The use of distance measures in phytosociological sampling. Ecology, 37: 451-460.

DOURMA M., 2011. Les forêts claires à Isoberlinia doka Craib \& Stapf et I. tomentosa (Harms) Craib \& Stapf (Fabaceae) en zone soudanienne du Togo : écologie, régénération naturelle et activités humaines. Acta Botanica Gallica, 158 (1) : 141-144.

DOURMA M., GUELLY A. K., KOKOU K., BATAWILA K., WALA K., BELLEFONTAINE R., AKPAGANA K., 2006. Multiplication par drageonnage d'Isoberlinia doka et I. tomentosa au sein des formations arborées du Nord-Togo. Bois et Forêt des Tropiques, 289 (3) : 49-57.

DOURMA M., WALA K., BELLEFONTAINE R., BATAWILA K., GUELLY A. K., AKPAGANA K., 2009a. Comparaison de l'utilisation des ressources forestières et de la régénération entre deux types de forêts claires à Isoberlinia au Togo. Bois et Forêt des Tropiques, 302 (4) : 5-19.

DOURMA M., BATAWILA K., WALA K., KOKOU K., GUELLY A. K., BELLEFONTAINE R., DE FOUCAULT B., AKPAGANA K., 2009b. Régénération naturelle des peuplements à Isoberlinia en zone soudanienne du Togo, Afrique de l'Ouest. Acta Botanica Gallica, 156 (3) : 415-425.

FABER-LANGENDOEN D., GENTRY A. H., 1991. The structure and diversity of rain forests at Bajo Calima, Chaco region, Western Colombia. Biotropica, 23 (1): 2-11.

FAO, 2001. Évaluation des ressources forestières mondiales 2000. Rome, Italie, Fao, Rapport principal, Étude Fao : Forêts $\mathrm{n}^{\circ} 140$.

FONTON N. H., YABI C. C., DAH-DOVONON J. Z., ADOKO F. K., DOTCHAMOU T., 2009. Modélisation du volume du fût d'arbre pour une gestion durable des écosystèmes forestiers soudaniens. Bois et Forêts des Tropiques, 300 (2) : 95-100.

FRONTIER S., PICHOD-VIALE D., 1991. Écosystèmes : structure, fonctionnement, évolution. Paris, France, Masson, coll. Écologie, 21, $392 \mathrm{p}$.

HILL M. O., GAUCH H. G., 1980. Detendred correspondance analysis: an improved ordination technique. Vegetatio, 42: 47-58.

HOUINATO M., 2001. Phytosociologie, écologie, production et capacité de charge des formations végétales pâturées dans la région des monts Kouffé (Bénin). Thèse, Université libre de Bruxelles, Belgique, $255 \mathrm{p}$.

HOUINATO M., SINSIN B., 2001. La pression agro-pastorale sur la zone riveraine de la Réserve de la Biosphère de la Pendjari. Tropicultura, 18 (2) : 112-117.

KENT M., COKER P., 1992. Vegetation description and analysis: a practical approach. Chichester, Royaume-Uni, John Wiley \& Sons, 363 p.

KERSHAW A. K., 1968. Classification and ordination of Nigerian savanna vegetation. Journal of Ecology, 56: 467-482.
KOUAMÉ N. F., 1998. Influence de l'exploitation forestière sur la végétation et la flore de la forêt classée du Haut-Sassandra (Centre-ouest de la Côte d'Ivoire). Thèse, Université de Cocody, Côte d'Ivoire, 227 p.

LYKKE A. M., 2000. Local perception of vegetation change and priorities for conservation of woody savanna vegetation in Senegal. Journal of Environmental Management, 59: 107-120.

OUÉDRAOGO A., 2006. Diversité et dynamique de la végétation ligneuse de la partie orientale du Burkina Faso. Thèse, Université de Ouagadougou, Burkina Faso, 195 p.

OUOBA P., 2006. Flore et végétation de la forêt classée de Niangoloko, Sud-Ouest du Burkina Faso. Thèse, Université de Ouagadougou, Burkina Faso, 140 p.

OOTERHOORN M., KAPELLE M., 2000. Vegetation structure and composition along an interior-edge-exterior gradient in Costa Rican montane cloud forest. Forest Ecology and Management, 126 (3): 291-307.

SINSIN B., 2000. Stratégie d'adaptation du système d'élevage bovin aux pénuries fourragères chez les Bétamaribé du Bénin. Berichte des Sonderforchungsbereichs, 268 (14) : 209-222.

SINSIN B., 2001. Life forms and species diversity of woodland associations in northern Benin. Systematics and Geography of Plants, 71(2): 873-88.

SOKPON N., BIAOU S. H., OUINSAVI C., HUNHYET O., 2006. Bases techniques pour une gestion durable des forêts claires du Nord-Bénin : rotation, diamètre minimal d'exploitabilité et régénération. Bois et Forêts des Tropiques, 287 (1) : 45-57.

SWAMINATHAN S. M., 1990. Foreword. In: Mc Nely J. A., Miller K. M., Reid W. V., Mittermeier R. A., Werner T. (éds). Conserving the world's biological diversity. Uicn, Wri, Conservation International, Wwf-US, World Bank, 174 p.

TENTE B., SINSIN B., 2002. Diversité et structure des formations arborescentes du secteur Perma-Toucountouna dans la chaîne de l'Atacora (Bénin). Études sur la Flore et la Végétation du Burkina Faso, 6 : 31-42.

TER BRAAK C. J. F., SMILAUER P., 1998. CANOCO reference manual and user's guide to CANOCO for Windows. Software for canonical community ordination (version 4). Ithaca, NY, États-Unis, Microcomputer Power, 352 p.

UBOM R. M., ISICHEI A. O., 1995. Soil vegetation interelationships in Isoberlinia woodlands in northwestern Nigeria. Acta Botanica Hungarica, 33 (3/4): 289-301.

WATSON L., DALLWITZ M. J., 1993. Les genres des légumineuses Caesalpinioïdeae et Swartzieae : description, identification et recherche documentaire. Version $29^{\mathrm{e}}$. http://biodiversity.uno.edu/delta/, 14 décembre 2000.

WHITE F., 1986. La végétation d’Afrique. Mémoire, carte de la végétation d'Afrique. Unesco/Aetfa/Unso.

WITKOWSKI E. T. F., O'CONNOR T. G., 1996. Topo-edaphic, floristic and physiognomic gradients of woody plants in a semi-arid African savanna woodland. Vegetatio, 124: 9-23. 